\title{
Holomorphic Lefschetz Formula for Manifolds with Boundary *†
}

\author{
A. Kytmanov $\$$ \\ S. Myslivets $\S$ \\ N. Tarkhanov
}

November 12, 2002

\begin{abstract}
The classical Lefschetz fixed point formula expresses the number of fixed points of a continuous map $f: M \rightarrow M$ in terms of the transformation induced by $f$ on the cohomology of $M$. In 1966 Atiyah and Bott extended this formula to elliptic complexes over a compact closed manifold. In particular, they presented a holomorphic Lefschetz formula for compact complex manifolds without boundary, a result, in the framework of algebraic geometry due to Eichler (1957) for holomorphic curves. On compact complex manifolds with boundary the Dolbeault complex is not elliptic, hence the Atiyah-Bott theory is no longer applicable. To get rid of the difficulties related to the boundary behaviour of the Dolbeault cohomology, Donelli and Fefferman (1986) derived a fixed point formula for the Bergman metric. The purpose of this paper is to present a holomorphic Lefschetz formula on a compact complex manifold with boundary.
\end{abstract}

\section{Introduction}

If $M$ is a closed manifold and $f: M \rightarrow M$ is a continuous map, then the Lefschetz number of $f$ is defined by

$$
L(f)=\sum_{i}(-1)^{i} \operatorname{tr}(H f)_{i},
$$

${ }^{*}$ AMS subject classification: primary: $32 \mathrm{~S} 50$; secondary: 58G10.

${ }^{\dagger}$ Key words and phrases: The Dolbeault complex, the Lefschetz number, fixed points.

¥Supported by the Deutsche Forschungsgemeinschaft and the RFFI grant 99-01-00790.

${ }^{\S}$ Supported by the Deutsche Forschungsgemeinschaft and the RFFI grant 99-01-00790. 
where $(H f)_{i}$ denotes the induced endomorphism in the cohomology with real coefficients $H^{i}(M, \mathbb{R})$ and tr the trace. In 1926 Lefschetz published his famous fixed point formula, cf. [Lef26], expressing this global characteristic of $f$ in case all fixed points of $f$ are isolated as the sum of local indices $\nu(p)$ at fixed points. These indices are map degrees of $1-f$ considered as a map between small spheres centered at the fixed points.

His argument is based on the intersection theory applied to the cycles $\Delta$ and $\Gamma_{f}$ representing the diagonal and the graph of $f$ in $M \times M$, respectively. Their homological intersection number corresponds via the Künneth formula and the Poincaré duality to the Lefschetz number, and a straightforward calculation yields the equality of the geometric intersection number and the sum of the local indices.

A few years later, considering simplicial maps of finite simplicial complexes, Hopf proved an alternating sum formula which by simplicial approximation lead to an alternative proof of the Lefschetz formula, cf. [Hop29].

There is a well-established relationship between cohomological traces of maps and their fixed point sets. This relationship comes from two results: first, the relation between the fixed points of a function and its trace as a composition operator on a space of functions; and second, the relation between traces on spaces in a complex and associated traces on the cohomology of the complex.

The classical fixed point theorem of Lefschetz [Lef26] is easily formulated in terms of the de Rham complex. Indeed, let $M$ be a closed compact smooth manifold of dimension $n$ and let $\bigwedge^{i}=\mathbb{C} \otimes_{\mathbb{R}} \bigwedge^{i} T^{*} M$ be the bundle of complexvalued exterior forms of degree $i$ over $M$. The exterior derivative $d$, if restricted to differential forms of degree $i$, provides a map $d_{i}: \mathcal{E}\left(\bigwedge^{i}\right) \rightarrow \mathcal{E}\left(\bigwedge^{i+1}\right)$ satisfying $d_{i+1} d_{i}=0$. The de Rham complex

$$
0 \longrightarrow \mathcal{E}\left(\bigwedge^{0}\right) \stackrel{d_{0}}{\longrightarrow} \mathcal{E}\left(\bigwedge^{1}\right) \stackrel{d_{1}}{\longrightarrow} \ldots \stackrel{d_{n-1}}{\longrightarrow} \mathcal{E}\left(\bigwedge^{n}\right) \longrightarrow 0
$$

is known to be Fredholm, its cohomology $H^{i}\left(\mathcal{E}\left(\bigwedge^{*}\right)\right)$ is isomorphic to the $i$ dimensional cohomology of the manifold $M$ with coefficients in $\mathbb{C}$, cf. de Rham [dR55]. Each smooth map $f: M \rightarrow M$ has a natural lift to the complex $\mathcal{E}\left(\bigwedge^{\prime}\right)$ given by the "pull-back" operator $f^{\sharp}$ on differential forms. In other words, $f^{\sharp}$ restricts to a family of maps $f_{i}^{\sharp}: \mathcal{E}\left(\bigwedge^{i}\right) \rightarrow \mathcal{E}\left(\bigwedge^{i}\right)$ commuting with the differential of $\mathcal{E}\left(\bigwedge^{\prime}\right)$, i.e., $d_{i} f_{i}^{\sharp}=f_{i+1}^{\sharp} d_{i}$. Hence it follows that $f^{\sharp}$ induces an endomorphism $\left(H f^{\sharp}\right)_{i}$ of the de Rham cohomology $H^{i}\left(\mathcal{E}\left(\bigwedge^{\cdot}\right)\right)$, for each $i=0,1, \ldots, n$. By the above, the Lefschetz number of $f$ is the alternating sum of the traces of $\left(H f^{\sharp}\right)_{i}, i=0,1, \ldots, n$. In particular, if $f$ is the identity map of $M$ then $L(f)$ coincides with the Euler characteristic of the manifold $M$. The Lefschetz theorem deals with a situation which is, in a sense, at the opposite extreme from the case of the identity map. 
In [AB67], Atiyah and Bott established an analogue of the Lefschetz fixed point formula for geometric endomorphisms of elliptic complexes over a smooth compact closed manifold $M$. The original proof of the formula in [AB67] can be considered as a generalisation of Hopf's argument. Its central point is again an alternating trace formula for endomorphisms of elliptic complexes given by pseudodifferential operators. To prove it, Atiyah and Bott made essential use of the structure of pseudodifferential operators introduced in [KN65]. To state their result, let

$$
0 \longrightarrow \mathcal{E}\left(V^{0}\right) \stackrel{d_{0}}{\longrightarrow} \mathcal{E}\left(V^{1}\right) \stackrel{d_{1}}{\longrightarrow} \ldots \stackrel{d_{N-1}}{\longrightarrow} \mathcal{E}\left(V^{N}\right) \longrightarrow 0
$$

be such a complex, where $V^{i}$ are complex vector bundles over $M$ and $d_{i}$ classical pseudodifferential operators of type $V^{i} \rightarrow V^{i+1}$ satisfying $d_{i+1} d_{i}=0$. The ellipticity of $\mathcal{E}\left(V^{\cdot}\right)$ means that the corresponding sequence of principal symbols

$$
0 \longrightarrow \pi^{*} V^{0} \stackrel{\sigma\left(d_{0}\right)}{\longrightarrow} \pi^{*} V^{1} \stackrel{\sigma\left(d_{1}\right)}{\longrightarrow} \ldots \stackrel{\sigma\left(d_{N-1}\right)}{\longrightarrow} \pi^{*} V^{N} \longrightarrow 0
$$

is exact in the complement of the zero section of $T^{*} M$. Here, $\pi^{*} V^{i} \rightarrow T^{*} M$ is the pull-back of $V^{i}$ under the canonical map $\pi: T^{*} M \rightarrow M$. Just as in the case of the de Rham complex, the cohomology $H^{i}\left(\mathcal{E}\left(V^{*}\right)\right)=\operatorname{ker} d_{i} / \operatorname{im} d_{i-1}$ of an elliptic complex is finite dimensional at each step $i$. Suppose $E$ is an endomorphism of the complex $\mathcal{E}\left(V^{\cdot}\right)$, i.e., a sequence $E_{i}: \mathcal{E}\left(V^{i}\right) \rightarrow \mathcal{E}\left(V^{i}\right)$ of linear maps such that $d_{i} E_{i}=E_{i+1} d_{i}$. Then $E$ preserves the spaces of cocycles and coboundaries of $\mathcal{E}\left(V^{\cdot}\right)$, hence after passing to quotient spaces it induces an endomorphism $(H E)_{i}$ of $H^{i}\left(\mathcal{E}\left(V^{\cdot}\right)\right)$ for all $i$. As these are finite dimensional, the traces $\operatorname{tr}(H E)_{i}$ are well defined which yields the Lefschetz number of $E$ by $L(E)=\sum_{i=0}^{N}(-1)^{i} \operatorname{tr}(H E)_{i}$. If $E=$ Id is the identity endomorphism of $\mathcal{E}\left(V^{\cdot}\right)$ then

$$
L(\mathrm{Id})=\sum_{i=0}^{N}(-1)^{i} \operatorname{dim} H^{i}\left(\mathcal{E}\left(V^{\cdot}\right)\right)
$$

is just the Euler characteristic $\chi\left(\mathcal{E}\left(V^{\cdot}\right)\right)$ of the complex $\mathcal{E}\left(V^{\cdot}\right)$. In particular, if $N=1$, this becomes the index of the elliptic operator $d_{0}$. The question of how to compute $L(E)$ is therefore a generalisation of the index problem for elliptic operators.

Atiyah and Bott [AB67] evaluated the Lefschetz number $L(E)$ in the case when $E$ is a geometric endomorphism of $\mathcal{E}\left(V^{\cdot}\right)$. The latter is constructed via a smooth map $f$ of the underlying manifold $M$ and a family of smooth bundle homomorphisms $h_{V^{i}}: f^{*} V^{i} \rightarrow V^{i}$. An endomorphism $E$ is said to be geometric if all $E_{i}$ are of the form $E_{i}=h_{V^{i}} \circ f^{*}$. Then, the Lefschetz formula of [AB67] reads

$$
L(E)=\sum_{f(p)=p} \frac{\sum_{i=0}^{N}(-1)^{i} \operatorname{tr} h_{V^{i}}(p)}{|\operatorname{det}(1-d f(p))|}
$$


provided that $f$ is of general position.

Note that the bundle homomorphism $h_{V^{i}}: f^{*} V^{i} \rightarrow V^{i}$ is just a family of linear maps $h_{V^{i}}(p): V_{f(p)}^{i} \rightarrow V_{p}^{i}$. Hence, at a fixed point $p$ of $f$ we have $V_{f(p)}^{i}=V_{p}^{i}$, and so $h_{V^{i}}(p)$ is an endomorphism of the vector space $V_{p}^{i}$. Thus, $\operatorname{tr} h_{V^{i}}(p)$ is defined.

In the case of the de Rham complex we have $h_{V^{i}}(p)=\bigwedge^{i} d f(p)^{\prime}$, the $i$ th exterior power of the transpose to $d f(p)$.

Thus, the Atiyah-Bott formula expresses the Lefschetz number of a geometric endomorphism of an elliptic complex on a closed compact manifold via infinitesimal invariants $\nu(p)$ of $f$ and $h_{V}$. at the fixed points of the map $f$. It is worth pointing out that formula (0.3) does not explicitly involve the pseudodifferential operators $d_{i}$. Thus it is much simpler than the AtiyahSinger index formula. Of course the $d_{i}$ are implicitly involved by the condition $d_{i} E_{i}=E_{i+1} d_{i}$.

In general the local index is a complex number and not an integer. The classical Lefschetz formula, where the local index is \pm 1 , is highly special in this direction. On the other hand the Lefschetz number $L(E)$ is a linear combination of traces and so, if $E$ is of finite order, $L(E)$ will be an algebraic integer. In these cases [AB67] leads to "integrability theorems" analogous to the integrability theorems obtained from the index theorem.

In [AB67] two more ways are sketched to prove the fixed point formula for elliptic complexes. The first approach relies on the results of Seeley [See67] on complex powers of pseudodifferential operators and the $\zeta$-function. A closely related approach was chosen by Kotake in [Kot69]. It consists of the study of the fundamental solution for the heat equation defined by the Laplacians of the complex after having transformed the Lefschetz number by means of the Hodge theory.

Taking up the second suggestion of Atiyah and Bott is what the paper of Nestke [Nes81] aims at. It runs along the lines of the original proof of Lefschetz for the classical formula.

It is natural to ask whether the local index $\nu(p)$ can be explained as a special case of a cohomological formula which always makes sense for isolated fixed points, as in the classical theorem where sign $\operatorname{det}(1-d f(p))=\operatorname{deg}(1-f, p)$. Toledo [Tol73] gave an exposition of the fixed point theory on a closed compact manifold which applied to isolated fixed points gives both the Atiyah-Bott formula and cohomological formulas. This method is based on a classical formula of de Rham [dR55, §33] which expresses intersection numbers in Riemannian manifolds in terms of the Green kernel.

A fixed point formula for higher-dimensional sets of fixed points was found by Gilkey in [Gil79] by means of heat equation methods.

In the ' 80 s the interest in the Atiyah-Bott formula increased enormously. This is first of all explained by the connection discovered between the index 
theorem and sypersymmetric quantum theories, cf. Alvarez-Gaumé [AG83] and Witten [Wit82]. There appeared new proofs of the Atiyah-Bott formula, cf. Atiyah and Segal [AS68], Bismut [Bis84, Bis85]. In the paper of Efremov [Efr88] the Atiyah-Bott fixed point formula is extended to universal coverings of a closed manifold. In the $L^{2}$-cohomology setting there are various further extensions of the Atiyah-Bott formula to non-compact manifolds, e.g., by Shubin [Shu92] and by Shubin and Seifarth [SS90]. A new idea suggested by Fedosov in [Fed93] is to consider endomorphisms of elliptic complexes which are induced by symplectic canonical transformations of $T^{*} M$ rather than by a map $f$ of the underlying manifold. A modern development of this idea actually leads to a Lefschetz fixed point theorem in deformation quantisation, cf. [Fed00].

Brenner and Shubin [BS81] proved an analogue of the Lefschetz fixed point formula for elliptic complexes on a compact smooth manifold with boundary $M$, whose differentials are operators in Boutet de Monvel's algebra, cf. [BdM71]. The ellipticity of $(0.1)$ on a manifold with boundary requires not only the exactness of (0.2) but also that of the corresponding sequence of boundary symbols

$$
\left.\left.\left.0 \longrightarrow \pi_{S}^{*} H_{+} \otimes V^{0}\right|_{S} \stackrel{\sigma_{\partial}\left(d_{0}\right)}{\longrightarrow} \pi_{S}^{*} H_{+} \otimes V^{1}\right|_{S} \stackrel{\sigma_{\partial}\left(d_{1}\right)}{\longrightarrow} \ldots \stackrel{\sigma_{\partial}\left(d_{N-1}\right)}{\longrightarrow} \pi_{S}^{*} H_{+} \otimes V^{N}\right|_{S} \longrightarrow 0
$$

where $S$ is the boundary of $M, H_{+}$the subspace of $\mathcal{S}^{\prime}(\mathbb{R})$ consisting of the Fourier transforms of all functions in $\chi_{[0, \infty)} \mathcal{S}(\mathbb{R})$, and $\pi_{S}: T^{*} S \rightarrow S$ the canonical projection. Any elliptic complex in this generalised sense is Fredholm, and so every its endomorphism $E$ has a well-defined Lefschetz number $L(E)$. Suppose that $E_{i}=h_{V^{i}} \circ f^{*}$ is a geometric endomorphism of (0.1) associated with a smooth map $f$ of the underlying manifold $M$. We need a specification of simple fixed points of $f$ on the boundary. Let $p$ be such a point, i.e., $p \in S$ and $f(p)=p$. Then $d f(p)$ induces a map $d_{S} f(p): T_{p} S \rightarrow T_{p} S$ and hence a map

$$
\left(d f / d_{S} f\right)(p): N_{p} S \rightarrow N_{p} S
$$

of the quotient space $N_{p} S=T_{p} M / T_{p} S$. The latter can be identified with the normal space to the boundary at the point $p$. As $N_{p} S$ is one-dimensional, (0.5) reduces to multiplication by a number $q(p) \in \mathbb{R}$. It is clear that $q(p) \geq 0$, for $M$ is invariant under $f$. Moreover, if $p$ is a simple fixed point of $f$, then $q(p) \neq 1$. A simple fixed point $p \in S$ is said to be attracting, if $q(p)<1$, and repulsing, if $q(p)>1$. Denote by $\operatorname{Fix}(f, M \backslash S)$ the set of all interior fixed points of $f$ and by $\operatorname{Fix}^{(a)}(f, S)$ the set of all attracting boundary fixed points of $f$. The main result of [BS81] states that if $f$ is a smooth map of $M$ with 
only simple fixed points then

$$
L(E)=\sum_{p \in \operatorname{Fix}(f, M \backslash S) \cup \mathrm{Fix}^{(a)}(f, S)} \frac{\sum_{i=0}^{N}(-1)^{i} \operatorname{tr} h_{V^{i}}(p)}{|\operatorname{det}(1-d f(p))|} .
$$

Note that if $M$ is a closed compact manifold, then the second term in the right side of (0.6) is absent and this equality becomes the Atiyah-Bott formula (cf. $(0.3))$.

The theorem of [BS81] applies to the de Rham complex on any smooth compact manifold with boundary, the local index being sign $\operatorname{det}(1-d f(p))$. In this case formula (0.6) can be deduced from the general Lefschetz fixed point theorem for CW-complexes, cf. Proposition 6.6 in [Dol72, Ch. 7]. However, this theorem gives no explicit description of the contribution of a non-interior fixed point while permitting $f$ with arbitrary fixed sets. We also mention the infinitesimal version of the classical Lefschetz formula for manifolds with boundary by Arnold [Arn79].

A particular case of (0.3) is the Lefschetz fixed point formula for the Dolbeault complex which is referred to as the holomorphic Lefschetz formula. For holomorphic curves $M$ this formula had already been established by Eichler in [Eic57]. For direct constructions along more classical lines we refer the reader to Patodi [Pat73], Toledo and Tong [TT75], Inoue [Ino82], et al. Donnelly and Fefferman [DF86] found an analogue of the holomorphic Lefschetz formula for strictly pseudoconvex domains in $\mathbb{C}^{n}$ provided with the Bergman metric. This corresponds to the case of a non-compact manifold.

At the very beginning of the ' $80 \mathrm{~s}$ M. Shubin called our attention to a fixed point theory on a compact complex manifold with boundary. Note that the results of [BS81] are not applicable to the Dolbeault complex on a complex manifold with boundary, for this complex is not elliptic. Although the cohomology of the Dolbeault complex is finite dimensional at steps $i>0$ for strictly pseudoconvex manifolds, the cohomology at step 0 is not. As but one difficulty caused by this we mention that of defining the Lefschetz number for complex manifolds with boundary. In the ' 80 s the problem was intensively treated by A. Brenner who also studied the fixed point theory for the tangential CauchyRiemann complex. However, his leaving for Israel and switching to computer science in the '90s didn't allow him to bring the investigations to a happy end, cf. [Bre88].

The purpose of this paper is to show a Lefschetz fixed point theorem for the Dolbeault complex on a strictly pseudoconvex compact manifold with boundary. The approach we take up is quite different from that of [BS81] and [Bre88] who followed in the large the scheme suggested in [AB67]. This would require a sophisticated technique of pseudodifferential operators on the Heisenberg 
group. Instead we make use of the explicit integral formulas for the Lefschetz number developed in [Tar95].

In order to formulate our result we let $M$ be a compact complex manifold of dimension $n>1$. The complex cotangent bundle then splits into a direct sum of complex subbundles $\mathbb{C} \otimes_{\mathbb{R}} T^{*} M=T^{1,0} M \oplus T^{0,1} M$ where $T^{1,0} M$ is spanned by the $d z_{j}$ and $T^{0,1} M$ by the $d \bar{z}_{k}$ of a local holomorphic coordinate system. Denote by $\bigwedge^{0, i}$ the bundle of exterior forms of bidegree $(0, i)$ over $M$. The exterior derivative $d$ decomposes correspondingly into a direct sum $d=\partial+\bar{\partial}$ where $\bar{\partial}_{i}: \mathcal{E}\left(\bigwedge^{0, i}\right) \rightarrow \mathcal{E}\left(\bigwedge^{0, i+1}\right)$ satisfies the integrability condition $\bar{\partial}^{2}=0$. The operator $\bar{\partial}$ acting on all of $\mathcal{E}\left(\bigwedge^{0, i}\right)$ is an elliptic operator. The complex $\mathcal{E}\left(\bigwedge^{0, \cdot}\right)$ is called the Dolbeault complex of the manifold $M$. It is elliptic in the interior of $M$, i.e., the sequence $(0.2)$ is exact away from the zero section of $T^{*} M$.

More generally if $V$ is any holomorphic vector bundle over $M$ the operator $1 \otimes \bar{\partial}$ is well defined on $\mathcal{E}\left(V \otimes \bigwedge^{0, \cdot}\right)$ and so determines a complex

$$
0 \longrightarrow \mathcal{E}\left(V \otimes \bigwedge^{0,0}\right) \stackrel{1 \otimes \bar{\partial}_{0}}{\longrightarrow} \mathcal{E}\left(V \otimes \bigwedge^{0,1}\right) \stackrel{1 \otimes \bar{\partial}_{1}}{\longrightarrow} \ldots \stackrel{1 \otimes \bar{\partial}_{n}-1}{\longrightarrow} \mathcal{E}\left(V \otimes \bigwedge^{0, n}\right) \longrightarrow 0
$$

which is elliptic in the interior of $M$. This complex is referred to as the Dolbeault complex with coefficients in $V$. A typical example is $V=\bigwedge^{p, 0}$, the bundle of exterior forms of bidegree $(p, 0)$ over $M$. Consider now a holomorphic map $f: M \rightarrow M$. The natural lifting of $f$ to $\bigwedge$ is then compatible with $\bar{\partial}$ and therefore induces an endomorphism $f^{0, \sharp}$ in the complex $\mathcal{E}\left(\bigwedge^{0, \cdot}\right)$. If $h: f^{*} V \rightarrow V$ is an arbitrary holomorphic bundle homomorphism then the tensor product $h \otimes \bigwedge^{i}(\partial f)^{*}$ serves to define the $i$ th lifting of $f$ to the complex $\mathcal{E}\left(V \otimes \bigwedge^{0, \cdot}\right)$. We write $E=h \otimes f^{0, \sharp}$ for the induced endomorphism of $\mathcal{E}\left(V \otimes \bigwedge^{0, \cdot}\right)$. Suppose $M$ is strictly pseudoconvex. Then the cohomology $H^{i}\left(\mathcal{E}\left(V \otimes \bigwedge^{0, \cdot}\right)\right)$ is known to be finite dimensional at all steps $i$ but $i=0$. The cohomology at step $i=0$ just amounts to the space of all $C^{\infty}$ sections of $V$ over $M$ which are holomorphic in the interior of $M$. We define the trace of $(H E)_{0}$ on $H^{0}\left(\mathcal{E}\left(V \otimes \bigwedge^{0, \cdot}\right)\right)$ by a special regularisation. Then the Lefschetz number of $E$ is introduced in the same way as above. We show that if $f$ is a smooth map of $M$ with only simple fixed points then

$$
L(E)=\sum_{p \in \operatorname{Fix}(f, M \backslash S) \cup \mathrm{Fix}^{(a)}(f, S)} \frac{\operatorname{tr} h(p)}{\operatorname{det}_{\mathbb{C}}(1-\partial f(p))} .
$$

Note that the right-hand side of (0.7) coincides with that of (0.6). In other words, the result of [BS81] still applies to the Dolbeault complex on a strictly pseudoconvex compact manifold, as if this complex were elliptic with respect to both sequences $(0.2)$ and (0.4).

From now on we restrict our attention to the case $V=M \times \mathbb{C}$. Clearly, this is the basic concept. The same proof still goes for general $V$. 


\section{Augmented Dolbeault complex}

Throughout the paper, we take $M=\overline{\mathcal{D}}$ where $\mathcal{D}$ is a strictly linearly convex domain in $\mathbb{C}^{n}, n>1$, with $C^{\infty}$ boundary. We write $\mathcal{D}=\left\{z \in \mathbb{C}^{n}: \varrho(z)<0\right\}$ where $\varrho$ is a $C^{\infty}$ real-valued function on $\mathbb{C}^{n}$ whose gradient does vanish on $\partial \mathcal{D}$. The strict linear convexity of $\mathcal{D}$ just amounts to saying that $\left\langle\nabla_{\zeta} \varrho, z-\zeta\right\rangle \neq 0$ for all $\zeta \in \partial \mathcal{D}$ and $z \in \overline{\mathcal{D}}$ with $z \neq \zeta$. In other words, the complex tangent hyperplane of $\partial \mathcal{D}$ at each point $\zeta \in \partial \mathcal{D}$ meets the closure of $\mathcal{D}$ at the only point $z=\zeta$.

Consider the Dolbeault complex

$$
0 \longrightarrow \mathcal{E}^{0}(\overline{\mathcal{D}}) \stackrel{\bar{\partial}}{\longrightarrow} \mathcal{E}^{1}(\overline{\mathcal{D}}) \stackrel{\bar{\partial}}{\longrightarrow} \ldots \stackrel{\bar{\partial}}{\longrightarrow} \mathcal{E}^{n}(\overline{\mathcal{D}}) \longrightarrow 0
$$

where $\mathcal{E}^{q}(\overline{\mathcal{D}})$ is the space of all differential forms of bidegree $(0, q)$ with $C^{\infty}$ coefficients in $\overline{\mathcal{D}}$. It is well known that the cohomology $H^{q}(\mathcal{E} \cdot(\overline{\mathcal{D}}))$ of $(1.1)$ is zero at every step $q>0$, cf. [Khe85, T. 8.12] and elsewhere. The cohomology $H^{0}(\mathcal{E} \cdot(\overline{\mathcal{D}}))$ at step 0 is still infinite dimensional. It coincides with the space $\mathcal{E}(\overline{\mathcal{D}}) \cap \mathcal{O}(\mathcal{D})$ of all $C^{\infty}$ functions in $\overline{\mathcal{D}}$ which are holomorphic in the interior of $\mathcal{D}$.

In the sequel we need also the augmented Dolbeault complex

$$
0 \longrightarrow \mathcal{E}(\overline{\mathcal{D}}) \cap \mathcal{O}(\mathcal{D}) \stackrel{\imath}{\longrightarrow} \mathcal{E}^{0}(\overline{\mathcal{D}}) \stackrel{\bar{\partial}}{\longrightarrow} \mathcal{E}^{1}(\overline{\mathcal{D}}) \stackrel{\bar{\partial}}{\longrightarrow} \ldots \stackrel{\bar{\partial}}{\longrightarrow} \mathcal{E}^{n}(\overline{\mathcal{D}}) \longrightarrow 0
$$

where $\imath$ is the embedding operator. The cohomology of (1.2) at each step $q \geq 1$ is equal to the corresponding cohomology of (1.1). On the other hand, the cohomology of (1.2) at steps -1 and 0 vanishes. Therefore, the complex (1.2) is Fredholm, cf. for instance [Hör85, §19.1], in contrast to the complex (1.1).

Consider a holomorphic map $f: \mathcal{D} \rightarrow \mathcal{D}$ which extends to a $C^{\infty}$ map of $\overline{\mathcal{D}}$. If in particular $f$ is proper then it automatically extends to a $C^{\infty}$ map of $\overline{\mathcal{D}}$, cf. Theorem 11 of [Pin86, Ch. 2]. In what follows we assume that $f$ has only isolated fixed points $f(z)=z$ in $\overline{\mathcal{D}}$. Then the number of such points in $\overline{\mathcal{D}}$ is finite.

The map $f$ induces the endomorphism $E=\left\{E_{q}\right\}$ of (1.2) given by $E_{q}=f_{q}^{\sharp}$, where

$$
f_{q}^{\sharp}: \mathcal{E}^{q}(\overline{\mathcal{D}}) \rightarrow \mathcal{E}^{q}(\overline{\mathcal{D}})
$$

is the "pull-back" operator on differential forms of bidegree $(0, q)$ under the map $f$. Since $f$ is holomorphic, $f_{0}^{\sharp}$ preserves also the space $\mathcal{E}(\overline{\mathcal{D}}) \cap \mathcal{O}(\mathcal{D})$. Denote by $H f^{\sharp}=\left\{\left(H f^{\sharp}\right)_{q}\right\}$ the corresponding endomorphism of the cohomology of (1.2). 
Definition 1.1 By the Lefschetz number of the augmented complex (1.2) is meant

$$
L_{\mathrm{p}}\left(f^{\sharp}\right)=\sum_{q=-1}^{n}(-1)^{q} \operatorname{tr}\left(H f^{\sharp}\right)_{q} .
$$

Here $\operatorname{tr}\left(H f^{\sharp}\right)_{q}$ is the trace of the endomorphism $\left(H f^{\sharp}\right)_{q}$ on the cohomology of (1.2) at step $q$, which is well defined. By the above, $\operatorname{tr}\left(H f^{\sharp}\right)_{q}$ vanishes for $q=-1$ and $q=0$. Hence $L_{\mathrm{p}}\left(f^{\sharp}\right)$ can be referred to as a partial Lefschetz number of the endomorphism $f^{\sharp}$ of (1.1). However, there is no canonical way to introduce a total Lefschetz number $L_{t}\left(f^{\sharp}\right)$ for this endomorphism of (1.1) because the cohomology $H^{0}(\mathcal{E} \cdot(\overline{\mathcal{D}}))$ is infinite dimensional. Hence, the trace of $\left(H f^{\sharp}\right)_{0}$ on $H^{0}(\mathcal{E} \cdot(\overline{\mathcal{D}}))$ requires a suitable regularisation. The aim of this paper is to properly define a total Lefschetz number for the endomorphism $f^{\sharp}$ of the Dolbeault complex and evaluate it in terms of infinitesimal invariants of fixed points of $f$.

\section{A parametrix of the Dolbeault complex}

Our calculations are based on an explicit fundamental solution of the $\bar{\partial}$ problem in strictly linearly convex domains of $\mathbb{C}^{n}$, cf. Theorem 8.9 in [Khe85] It allows one to construct an explicit fundamental solution of the augmented complex (1.2).

To describe this construction, set

$$
\begin{aligned}
P(\zeta) & =\nabla_{\zeta} \\
& =\left(\frac{\partial \varrho}{\partial \zeta_{1}}, \ldots, \frac{\partial \varrho}{\partial \zeta_{n}}\right)
\end{aligned}
$$

and

$$
\begin{aligned}
\Phi(\zeta, z) & =\langle P(\zeta), \zeta-z\rangle \\
& =\sum_{j=1}^{n} \frac{\partial \varrho}{\partial \zeta_{j}}\left(\zeta_{j}-z_{j}\right) .
\end{aligned}
$$

The function $\Phi(\zeta, z)$ is different from zero for all $\zeta \in \partial \mathcal{D}$ and $z \in \overline{\mathcal{D}}$ with $\zeta \neq z$, for $\mathcal{D}$ is strictly linearly convex.

Given any smooth function $\eta=\eta(\zeta, z, \lambda)$ of $(\zeta, z, \lambda) \in \mathbb{C}^{n} \times \mathbb{C}^{n} \times \mathbb{R}$ with values in $\mathbb{C}^{n}$, define a Leray form

$$
\omega^{\prime}(\eta)=\sum_{j=1}^{n}(-1)^{j-1} \eta_{j} d \eta[j]
$$


where $d \eta[j]$ is the wedge product of the differentials $d \eta_{1}, \ldots, d \eta_{n}$ one after another excepting $d \eta_{j}$. Set also $d \eta=d \eta_{1} \wedge \ldots \wedge d \eta_{n}$. Then we can certainly write

$$
\left(\omega^{\prime}(\eta)\right) \wedge d \zeta \wedge d z=\left(\sum_{q=0}^{n} \omega_{q}^{\prime}(\eta)\right) \wedge d \zeta \wedge d z,
$$

with $\omega_{q}^{\prime}(\eta)$ a differential form of degree $n-q-1$ in $d \bar{\zeta}_{1}, \ldots, d \bar{\zeta}_{n}$ and $d \lambda$ and of degree $q$ in $d \bar{z}_{1}, \ldots, d \bar{z}_{n}$.

After [Khe85, §8] we introduce integral operators

$$
\begin{aligned}
& \left(T_{q} u\right)(z)=(-1)^{q} \int_{\mathcal{D}} u(\zeta) \wedge \frac{(n-1) !}{(2 \pi \imath)^{n}} \omega_{q-1}^{\prime}\left(\frac{\bar{\zeta}-\bar{z}}{|\zeta-z|^{2}}\right) \wedge d \zeta \\
& \left(L_{q} u\right)(z)=(-1)^{q} \int_{\partial \mathcal{D} \times[0,1]} u(\zeta) \wedge \frac{(n-1) !}{(2 \pi \imath)^{n}} \omega_{q-1}^{\prime}\left((1-\lambda) \frac{\bar{\zeta}-\bar{z}}{|\zeta-z|^{2}}+\lambda \frac{P(\zeta)}{\Phi(\zeta, z)}\right) \wedge d \zeta
\end{aligned}
$$

for $u \in \mathcal{E}^{q}(\overline{\mathcal{D}})$, where

$$
\begin{aligned}
& (1-\lambda) \frac{\bar{\zeta}-\bar{z}}{|\zeta-z|^{2}}+\lambda \frac{P(\zeta)}{\Phi(\zeta, z)} \\
& \quad=\left((1-\lambda) \frac{\bar{\zeta}_{1}-\bar{z}_{1}}{|\zeta-z|^{2}}+\lambda \frac{P_{1}(\zeta)}{\Phi(\zeta, z)}, \ldots,(1-\lambda) \frac{\bar{\zeta}_{n}-\bar{z}_{n}}{|\zeta-z|^{2}}+\lambda \frac{P_{n}(\zeta)}{\Phi(\zeta, z)}\right)
\end{aligned}
$$

for $0 \leq \lambda \leq 1$.

The operators $T_{q}$ and $L_{q}$ are known to map $\mathcal{E}^{q}(\overline{\mathcal{D}} \cap U)$ to $\mathcal{E}^{q-1}(\overline{\mathcal{D}} \cap U)$. Denote

$$
\begin{array}{lll}
P_{q}=T_{q}+L_{q} & \text { if } & 1 \leq q \leq n, \\
P_{q}=0 & \text { if } \quad q=0 \text { or } q=n+1 .
\end{array}
$$

For an arbitrary differential form $u \in \mathcal{E}^{q}(\overline{\mathcal{D}})$, we define also a CauchyFantappiè integral

$$
\left(F_{q} u\right)(z)=(-1)^{q} \int_{\partial \mathcal{D}} u(\zeta) \wedge \frac{(n-1) !}{(2 \pi \imath)^{n}} \omega_{q}^{\prime}\left(\frac{P(\zeta)}{\Phi(\zeta, z)}\right) \wedge d \zeta, \quad z \in \mathcal{D} .
$$

These operators are connected with each other by the well-known integral formulas of Leray-Koppelman.

Lemma 2.1 Any differential form $u \in \mathcal{E}^{q}(\overline{\mathcal{D}}), q \geq 0$, can be represented in $\mathcal{D}$ by

$$
u=F_{q} u+P_{q+1}(\bar{\partial} u)+\bar{\partial}\left(P_{q} u\right) .
$$

Proof. By the formula of Koppelman, we get

$$
u=M_{q} u+T_{q+1}(\bar{\partial} u)+\bar{\partial}\left(T_{q} u\right)
$$


in $\mathcal{D}$, where

$$
\left(M_{q} u\right)(z)=(-1)^{q} \int_{\partial \mathcal{D}} u(\zeta) \wedge \frac{(n-1) !}{(2 \pi \imath)^{n}} \omega_{q}^{\prime}\left(\frac{\bar{\zeta}-\bar{z}}{|\zeta-z|^{2}}\right) \wedge d \zeta
$$

cf. [AD83]. On the other hand, the identity $\left(\bar{\partial}_{\zeta}+d_{\lambda}\right) \omega_{q}^{\prime}(\eta)+\bar{\partial}_{z} \omega_{q-1}^{\prime}(\eta)=0$ implies that

$$
M_{q} u-F_{q} u=L_{q+1}(\bar{\partial} u)+\bar{\partial}\left(L_{q} u\right)
$$

in the domain $\mathcal{D}$. Combining these two equalities, we arrive at the desired formula (2.5).

The integral $F_{q} u$ actually vanishes for $q>0$ since

$$
\frac{P(\zeta)}{\Phi(\zeta, z)}
$$

is holomorphic in the variable $z$.

Since the kernel of $F_{0}$ is holomorphic in the exterior variable $z$, the operator $F_{0}$ maps $\mathcal{E}(\overline{\mathcal{D}})$ to $\mathcal{E}(\overline{\mathcal{D}}) \cap \mathcal{O}(\mathcal{D})$. Hence the following lemma says that the operators $\left\{F_{0}, P_{1}, \ldots, P_{n}\right\}$ constitute a fundamental solution of the augmented Dolbeault complex (1.2).

Lemma 2.2 As defined above, the operators $\left\{P_{q}\right\}$ and $F_{0}$ satisfy

$$
\begin{array}{rlrlrl}
F_{0} \imath u & =u-S_{-1} u & \text { for all } & u \in \mathcal{E}(\overline{\mathcal{D}}) \cap \mathcal{O}(\mathcal{D}), & & q=-1 ; \\
P_{1} \bar{\partial} u+\imath F_{0} u & =u-S_{0} u & \text { for all } u \in \mathcal{E}(\overline{\mathcal{D}}), & & q=0 ; \\
P_{q+1} \bar{\partial} u+\bar{\partial} P_{q} u & =u-S_{q} u & \text { for all } u \in \mathcal{E}^{q}(\overline{\mathcal{D}}), & & q>0,
\end{array}
$$

where $S_{-1}=0$.

Proof. Since $P_{0}=0$, all the equalities (2.7) follow from Lemma 2.1 even with $S_{q}=0$ for all $q$.

\section{Lefschetz number}

Note that all the operators $S_{q}$ in $(2.7)$ are smoothing, i.e., they map $\left(\mathcal{E}^{n-q}(\overline{\mathcal{D}})\right)^{\prime}$ to $\mathcal{E}^{q}(\overline{\mathcal{D}})$.

Lemma 3.1 The composition $f^{\sharp} \circ S$ is an endomorphism of the augmented Dolbeault complex, and

$$
L_{\mathrm{p}}\left(f^{\sharp}\right)=L_{\mathrm{p}}\left(f^{\sharp} \circ S\right) .
$$


Proof. Applying the endomorphism $f^{\sharp}$ to both sides of equalities (2.7) we readily obtain

$$
\begin{aligned}
\left(f_{-1}^{\sharp} \circ F_{0}\right) \imath & =f_{-1}^{\sharp}-f_{-1}^{\sharp} \circ S_{-1}, \\
\left(f_{0}^{\sharp} \circ P_{1}\right) \bar{\partial}+\imath\left(f_{-1}^{\sharp} \circ F_{0}\right) & =f_{0}^{\sharp}-f_{0}^{\sharp} \circ S_{0}, \\
\left(f_{q}^{\sharp} \circ P_{q+1}\right) \bar{\partial}+\bar{\partial}\left(f_{q-1}^{\sharp} P_{q}\right) & =f_{q}^{\sharp}-f_{q}^{\sharp} \circ S_{q}
\end{aligned}
$$

for all $q>0$. Since $f_{-1}^{\sharp} \circ F_{0}$ maps $\mathcal{E}^{0}(\overline{\mathcal{D}})$ to $\mathcal{E}(\overline{\mathcal{D}}) \cap \mathcal{O}(\mathcal{D})$ and $f_{q-1}^{\sharp} \circ P_{q}$ maps $\mathcal{E}^{q}(\overline{\mathcal{D}})$ to $\mathcal{E}^{q-1}(\overline{\mathcal{D}})$ for $q>0$, we deduce that $f^{\sharp}$ and $f^{\sharp} \circ S$ are homotopic endomorphisms of the augmented Dolbeault complex. Hence they induce the same endomorphism of the cohomology of (1.2), i.e., $H f^{\sharp}=H\left(f^{\sharp} \circ S\right)$, and the lemma follows.

Note that $f^{\sharp} \circ S$ is a smoothing endomorphism of the augmented Dolbeault complex (1.2).

Lemma 3.2 If $E=\left\{E_{q}\right\}_{q=-1}^{n}$ is a smoothing endomorphism of the complex (1.2), then

$$
L_{\mathrm{p}}(E)=\sum_{q=-1}^{n}(-1)^{q} \operatorname{tr} E_{q} .
$$

Proof. The proof of this lemma repeats the proofs of Lemmas 4.2, 4.3 and their Corollary in [BS91] word for word. It relies essentially on Theorem 19.1.15 of [Hör85]. Namely, consider Hilbert completions $H^{s q}(\mathcal{D})$ of order $s \geq 1$ of the spaces $\mathcal{E}^{q}(\overline{\mathcal{D}})$. The operators $E_{q}$ extend by continuity to maps of $H^{s q}(\mathcal{D})$, i.e., $E_{q}^{(s)}: H^{s q}(\mathcal{D}) \rightarrow H^{s q}(\mathcal{D})$. The extensions obtained this way are of trace class, and their traces are independent of $s$. Since the augmented Dolbeault complex (1.2) possesses a "good" parametrix given by Lemma 2.2, it follows that the cohomology of (1.2) is isomorphic to the cohomology of the augmented Dolbeault complex evaluated in Hilbert spaces $H^{s q}(\mathcal{D})$ in the usual way. Applying Theorem 19.1.15 of [Hör85] then establishes the generalised Euler formula (3.1).

\section{Integral formula for the Lefschetz number}

Denote by $K_{T_{q}}(\zeta, z)$ and $K_{L_{q}}(\zeta, z)$ the kernels of operators $T_{q}$ and $L_{q}$, respectively. Away from the diagonals of $\mathcal{D}$ and $\partial \mathcal{D}$ these are differential forms of bidegree $(n, n-q)$ in $\zeta$ and $(0, q)$ in $z$, and $(n, n-q-1)$ in $\zeta$ and $(0, q)$ in $z$, respectively. 
Let $\Delta$ stand for the diagonal map $\overline{\mathcal{D}} \rightarrow \overline{\mathcal{D}} \times \overline{\mathcal{D}}$. Then the operation $\operatorname{tr} \Delta^{*}$ is the usual restriction of a $2 n$-form on $\overline{\mathcal{D}} \times \overline{\mathcal{D}}$ to a $2 n$-form on $\overline{\mathcal{D}}$, i.e., $\operatorname{tr} \Delta^{*}=\Delta^{\sharp}$ with tr a trace in fibres.

Consider the following differential form of bidegree $(n, n-1)$ defined away from the set $\operatorname{Fix}(f, \overline{\mathcal{D}})$ in $\overline{\mathcal{D}}$ :

$$
\varphi(T)=\sum_{q=1}^{n}(-1)^{q} \Delta^{\sharp}(1 \times f)^{\sharp} K_{T_{q}} .
$$

Lemma 4.1 The Lefschetz number for the augmented Dolbeault complex is given by

$$
L_{\mathrm{p}}\left(f^{\sharp}\right)=\text { p.v. } \int_{\mathcal{D}} \Delta^{\sharp} \bar{\partial}_{\zeta}^{\prime}(1 \times f)^{\sharp} K_{T_{1}}-\text { p.v. } \int_{\mathcal{D}} d \varphi(T),
$$

where the integral is understood in the sense of principal value.

Proof. Since $f^{\sharp} \circ S$ is a smoothing endomorphism of (1.2) it follows from Lemmas 3.1 and 3.2 that

$$
\begin{aligned}
L_{\mathrm{p}}\left(f^{\sharp}\right) & =\sum_{q=1}^{n}(-1)^{q} \operatorname{tr} f_{q}^{\sharp} \circ S_{q} \\
& =\int_{\mathcal{D}} \sum_{q=1}^{n}(-1)^{q} \Delta^{\sharp}(1 \times f)^{\sharp} K_{S_{q}} .
\end{aligned}
$$

By assumption, the set $\operatorname{Fix}(f, \overline{\mathcal{D}})$ is of zero measure. Since the integrand in (4.3) is of class $L^{1}(\mathcal{D})$, we get

$$
L_{\mathrm{p}}\left(f^{\sharp}\right)=\lim _{\varepsilon \rightarrow 0} \int_{\mathcal{D} \backslash U_{\varepsilon}} \sum_{q=1}^{n}(-1)^{q} \Delta^{\sharp}(1 \times f)^{\sharp} K_{S_{q}}
$$

where $U_{\varepsilon}$ is the set of all points $\zeta \in \mathcal{D}$ whose distance to $\operatorname{Fix}(f, \overline{\mathcal{D}})$ is less than $\varepsilon$.

We now make use of equalities (2.7) to evaluate the integrand in the latter integral. Suppose $(\zeta, z) \in(\mathcal{D} \times \mathcal{D}) \backslash \Delta$ is an arbitrary point. Pick a differential form $u \in \mathcal{E}^{q}(\overline{\mathcal{D}})$ of degree $q \geq 0$, which is supported in a small ball around $\zeta$ and vanishes near $z$. Since the singularities of $K_{T_{q}}$ lie on $\Delta$ we obtain by Stokes' Theorem

$$
\begin{aligned}
P_{q+1} \bar{\partial} u+\bar{\partial} P_{q} u & =T_{q+1} \bar{\partial} u+\bar{\partial} T_{q} u \\
& =\int_{\mathcal{D}} u(\zeta) \wedge\left(\bar{\partial}_{\zeta}^{\prime} K_{T_{q+1}}(\zeta, z)+\bar{\partial}_{z} K_{T_{q}}(\zeta, z)\right) \\
& =-\int_{\mathcal{D}} u(\zeta) \wedge K_{S_{q}}(\zeta, z),
\end{aligned}
$$


where $\bar{\partial}_{\zeta}^{\prime}=(-1)^{q-1} \bar{\partial}_{\zeta}$ is the transpose of $\bar{\partial}$ on forms of bidegree $(0, q)$. It follows that

$$
-K_{S_{q}}=\bar{\partial}_{\zeta}^{\prime} K_{T_{q+1}}+\bar{\partial}_{z} K_{T_{q}}
$$

holds on $\mathcal{D} \backslash U_{\varepsilon}$, whence

$$
\begin{aligned}
\int_{\mathcal{D} \backslash U_{\varepsilon}} & \sum_{q=1}^{n}(-1)^{q} \Delta^{\sharp}(1 \times f)^{\sharp} K_{S_{q}} \\
= & -\int_{\mathcal{D} \backslash U_{\varepsilon}} \sum_{q=1}^{n}(-1)^{q} \Delta^{\sharp}\left(\bar{\partial}_{z}(1 \times f)^{\sharp} K_{T_{q}}-\bar{\partial}_{\zeta}^{\prime}(1 \times f)^{\sharp} K_{T_{q}}\right)+\Delta^{\sharp} \bar{\partial}_{\zeta}^{\prime}(1 \times f)^{\sharp} K_{T_{1}} \\
= & -\int_{\mathcal{D} \backslash U_{\varepsilon}} \bar{\partial}\left(\sum_{q=1}^{n}(-1)^{q} \Delta^{\sharp}(1 \times f)^{\sharp} K_{T_{q}}\right)+\Delta^{\sharp} \bar{\partial}_{\zeta}^{\prime}(1 \times f)^{\sharp} K_{T_{1}} .
\end{aligned}
$$

The differential form in parentheses is just $\varphi(T)$. Since it is of bidegree $(n, n-1)$ we may replace $\bar{\partial}$ by the total exterior derivative $d$, which establishes the lemma.

For $T_{q}$ given by (2.2) it follows from (2.1) that

$$
\begin{aligned}
\varphi(T) & =\Delta^{\sharp}(1 \times f)^{\sharp} \sum_{q=1}^{n}(-1)^{q} K_{T_{q}} \\
& =\Delta^{\sharp}(1 \times f)^{\sharp} \sum_{q=1}^{n} \frac{(n-1) !}{(2 \pi \imath)^{n}} \omega_{q-1}^{\prime}\left(\frac{\bar{\zeta}-\bar{z}}{|\zeta-z|^{2}}\right) \wedge d \zeta \\
& =\frac{(n-1) !}{(2 \pi \imath)^{n}} \omega^{\prime}\left(\frac{\overline{\zeta-f(\zeta)}}{|\zeta-f(\zeta)|^{2}}\right) \wedge d \zeta \\
& =\frac{(n-1) !}{(2 \pi \imath)^{n}} \sum_{j=1}^{n}(-1)^{j-1} \frac{\overline{\zeta_{j}-f_{j}(\zeta)}}{|\zeta-f(\zeta)|^{2 n}} d \zeta \wedge d \overline{(\zeta-f(\zeta))}[j],
\end{aligned}
$$

the form of incomplete logarithmic residue.

As mentioned, the endomorphism $f^{\sharp}$ on holomorphic functions in $\mathcal{D}$ which are $C^{\infty}$ up to boundary has no regular trace, for this space is infinite dimensional. Moreover, there are no canonical regularisation of the trace functional. Still we may introduce a regularised trace on holomorphic functions from purely heuristic considerations. On smooth functions $f^{\sharp}$ has the kernel $\delta(\zeta-f(z))$, hence its trace is

$$
\int_{\mathcal{D}} \delta(\zeta-f(\zeta)) d v
$$

where $v=(2 \imath)^{-n} d \bar{\zeta} \wedge d \zeta$ is the volume element of $\mathcal{D}$. On holomorphic functions in $\mathcal{D}$ smooth up to the boundary the Dirac functional fails to have a unique 
representation. Any integral representation of holomorphic functions can be actually thought of as a representation of the delta-function. The most suitable of them are integral representations whose kernels are holomorphic in the external variable $z$, for they determine projections onto the space of holomorphic functions. This is just the case for the component $F_{0}$ of the parametrix (2.7).

Definition $4.2 B y$ the regularised trace of the endomorphism $\left(H f^{\sharp}\right)_{0}$ on $H^{0}(\mathcal{E} \cdot(\overline{\mathcal{D}}))$ is meant

$$
\tilde{\operatorname{tr}}\left(H f^{\sharp}\right)_{0}=\text { p.v. } \int_{\partial \mathcal{D}} \Delta^{\sharp}(1 \times f)^{\sharp} K_{F_{0}}+\int_{\mathcal{D}} \Delta^{\sharp}(1 \times f)^{\sharp} K_{S_{0}} .
$$

Now we are in a position to introduce the total Lefschetz number $L_{\mathrm{t}}\left(f^{\sharp}\right)$ for the endomorphism $f^{*}$ of the Dolbeault complex (1.1) itself. Namely, let us set

$$
L_{\mathrm{t}}\left(f^{\sharp}\right)=\widetilde{\operatorname{tr}}\left(H f^{\sharp}\right)_{0}+L_{\mathrm{p}}\left(f^{\sharp}\right) .
$$

Lemma 4.3 As defined in (4.4), it follows that

$$
L_{\mathrm{t}}\left(f^{\sharp}\right)=\text { p.v. } \int_{\partial \mathcal{D}} \Delta^{\sharp}(1 \times f)^{\sharp} K_{F_{0}}-\text { p.v. } \int_{\mathcal{D}} d \varphi(T) .
$$

Proof. Indeed, analysis similar to that in the proof of Lemma 4.1 shows that

$$
\Delta^{\sharp}(1 \times f)^{\sharp} K_{S_{0}}=-\Delta^{\sharp} \bar{\partial}_{\zeta}^{\prime}(1 \times f)^{\sharp} K_{T_{1}}
$$

away from the set of fixed points of $f$. Hence the lemma follows.

\section{$5 \quad$ Local indices}

Suppose $\operatorname{Fix}(f, \overline{\mathcal{D}})$ consists of isolated fixed points only. For any $p \in \operatorname{Fix}(f, \overline{\mathcal{D}})$, denote by $B(p, \varepsilon)$ the ball of radius $\varepsilon>0$ with centre at $p$. If $\varepsilon$ is small enough then the ball $B(p, \varepsilon)$ does not contain any fixed point of $f$ different from $p$. Set

$$
U_{\varepsilon}=\left(\cup_{p \in \operatorname{Fix}(f, \overline{\mathcal{D}})} B(p, \varepsilon)\right) \cap \mathcal{D} .
$$

Using Lemma 4.1 we get by Stokes' formula

$$
\begin{aligned}
- \text { p.v. } \int_{\mathcal{D}} d \varphi(T) & =-\lim _{\varepsilon \rightarrow 0} \int_{\mathcal{D} \backslash U_{\varepsilon}} d \varphi(T) \\
& =-\lim _{\varepsilon \rightarrow 0} \int_{\partial \mathcal{D} \backslash U_{\varepsilon}} \varphi(T)+\sum_{p \in \operatorname{Fix}(f, \overline{\mathcal{D}})} \lim _{\varepsilon \rightarrow 0} \int_{\partial B(p, \varepsilon) \cap \mathcal{D}} \varphi(T) \\
& =- \text { p.v. } \int_{\partial \mathcal{D}} \varphi(T)+\sum_{p \in \operatorname{Fix}(f, \overline{\mathcal{D}})} \mu_{i}(p)
\end{aligned}
$$


where

$$
\mu_{i}(p)=\lim _{\varepsilon \rightarrow 0} \int_{\partial B(p, \varepsilon) \cap \mathcal{D}} \varphi(T)
$$

is called the local index of the fixed point $p$.

Lemma 5.1 If $p \in \mathcal{D}$ is a simple fixed point of $f$, i.e., $\operatorname{det}_{\mathbb{C}}\left(1-f^{\prime}(p)\right) \neq 0$, then

$$
\mu_{i}(p)=\frac{1}{\operatorname{det}_{\mathbb{C}}\left(1-f^{\prime}(p)\right)}
$$

Proof. We change the variables in a small neighbourhood of $p$ by the formula $w=\zeta-f(\zeta)$. Since $p$ is a simple fixed point of $f$ this change of variables is biholomorphic near $p$. Hence we obtain by the Bochner-Martinelli formula

$$
\begin{aligned}
\mu_{i}(p) & =\lim _{\varepsilon \rightarrow 0} \int_{(1-f) * \partial B(p, \varepsilon)} \frac{1}{\operatorname{det}_{\mathbb{C}} \frac{\partial w}{\partial \zeta}(\zeta(w))} \frac{(n-1) !}{(2 \pi \imath)^{n}} \omega^{\prime}\left(\frac{\bar{w}}{|w|^{2}}\right) \wedge d w \\
& =\lim _{\delta \rightarrow 0} \int_{\partial B(0, \delta)} \frac{1}{\operatorname{det}_{\mathbb{C}}\left(1-f^{\prime}(\zeta(w))\right)} \frac{(n-1) !}{(2 \pi \imath)^{n}} \sum_{j=1}^{n}(-1)^{j-1} \frac{\overline{w_{j}}}{|w|^{2 n}} d w \wedge d \bar{w}[j] \\
& =\frac{1}{\operatorname{det}_{\mathbb{C}}\left(1-f^{\prime}(p)\right)}
\end{aligned}
$$

as desired.

Note that the differential form $\varphi(T)$ is actually closed in a punctured neighbourhood of $a$. Hence the problem of evaluating $\mu_{i}(p)$ reduces in general to calculation of the so-called Grothendieck residues. In [Tsi92, §6] an algebraic interpretation of the local residue is given in terms of the trace of a finite extension (connected with the map $f$ ) of the field of germs of meromorphic functions at $p$.

For fixed points of $f$ on the boundary the local index $\mu_{i}(p)$ is still independent of whether $p$ is attracting or repulsing.

Lemma 5.2 If $p \in \partial \mathcal{D}$ is a simple fixed point of $f$ then

$$
\mu_{i}(p)=\frac{1}{2} \frac{1}{\operatorname{det}_{\mathbb{C}}\left(1-f^{\prime}(p)\right)} .
$$

Proof. Once again we make the change of variables $w=\zeta-f(\zeta)$ in a small neighbourhood of $p$. This yields

$$
\begin{aligned}
\mu_{i}(p) & =\lim _{\varepsilon \rightarrow 0} \int_{(1-f)_{*}(\partial B(p, \varepsilon) \cap \mathcal{D})} \operatorname{det}_{\mathbb{C}} \frac{\partial \zeta}{\partial w} \frac{(n-1) !}{(2 \pi \imath)^{n}} \omega^{\prime}\left(\frac{\bar{w}}{|w|^{2}}\right) \wedge d w \\
& =\frac{1}{\operatorname{det}_{\mathbb{C}}\left(1-f^{\prime}(p)\right)} \lim _{\varepsilon \rightarrow 0} \int_{(1-f)_{*}(\partial B(p, \varepsilon) \cap \mathcal{D})} \frac{(n-1) !}{(2 \pi \imath)^{n}} \omega^{\prime}\left(\frac{\bar{w}}{|w|^{2}}\right) \wedge d w
\end{aligned}
$$


for $\operatorname{det}_{\mathbb{C}} \zeta^{\prime}(w)$ is smooth at $w=0$. The holomorphic map $w=\zeta-f(\zeta)$ keeps the orientation of $\mathbb{C}^{n}$, hence the limit on the right is independent of whether $p$ is attracting or repulsing. In fact this limit is known to be $1 / 2$, which establishes the formula.

\section{Holomorphic Lefschetz formula}

We now come back to evaluating the total Lefschetz number (4.4) for the endomorphism $f^{*}$ of the Dolbeault complex (1.1). Namely, using (5.1) we easily get

$$
\begin{aligned}
L_{\mathrm{t}}\left(f^{\sharp}\right) & =\text { p.v. } \int_{\partial \mathcal{D}} \Delta^{\sharp}(1 \times f)^{\sharp} K_{F_{0}}-\text { p.v. } \int_{\partial \mathcal{D}} \varphi(T)+\sum_{p \in \operatorname{Fix}(f, \overline{\mathcal{D}})} \mu_{i}(p) \\
& =\text { p.v. } \int_{\partial \mathcal{D}} \Delta^{\sharp}(1 \times f)^{\sharp}\left(K_{F_{0}}-\sum_{q=1}^{n}(-1)^{q} K_{T_{q}}\right)+\sum_{p \in \operatorname{Fix}(f, \overline{\mathcal{D}})} \mu_{i}(p) .
\end{aligned}
$$

As already noted, the kernels $K_{F_{q}}$ vanish for $q>0$. On the other hand, the kernel $K_{T_{q}}$, if restricted to the boundary of $\mathcal{D}$, coincides with $-K_{M_{q-1}}$. Hence it follows that

$$
\begin{aligned}
\Delta^{\sharp}(1 \times f)^{\sharp}\left(K_{F_{0}}-\sum_{q=1}^{n}(-1)^{q} K_{T_{q}}\right) & =\Delta^{\sharp}(1 \times f)^{\sharp}\left(K_{F_{0}}-\sum_{q=1}^{n}(-1)^{q-1} K_{M_{q-1}}\right) \\
& =-\Delta^{\sharp}(1 \times f)^{\sharp} \sum_{q=0}^{n-1}(-1)^{q}\left(K_{M_{q}}-K_{F_{q}}\right)
\end{aligned}
$$

away from the set $\operatorname{Fix}(f, \partial \mathcal{D})$ on the boundary. Since $K_{M_{q}}-K_{F_{q}}$ is a difference of two Leray forms it is in fact exact. Its primitive can be easily calculated, namely

$$
\begin{aligned}
& \Delta^{\sharp}(1 \times f)^{\sharp} \sum_{q=0}^{n-1}(-1)^{q}\left(K_{M_{q}}-K_{F_{q}}\right) \\
& =\Delta^{\sharp}(1 \times f)^{\sharp} \sum_{q=0}^{n-1}(-1)^{q} K_{L_{q+1} \circ \bar{\partial}+\bar{\partial} \circ L_{q}} \\
& =\sum_{q=1}^{n}(-1)^{q} \Delta^{\sharp}(1 \times f)^{\sharp}\left(-\bar{\partial}_{\zeta}^{\prime} K_{L_{q}}+\bar{\partial}_{z} K_{L_{q}}\right) \\
& =\bar{\partial}\left(\sum_{q=1}^{n}(-1)^{q} \Delta^{\sharp}(1 \times f)^{\sharp} K_{L_{q}}\right),
\end{aligned}
$$


the first equality being due to (2.6). We can here replace the $\bar{\partial}$-derivative by the total derivative $d$, for the differential form

$$
\varphi(L)=\Delta^{\sharp}(1 \times f)^{\sharp} \sum_{q=1}^{n}(-1)^{q} K_{L_{q}}
$$

is of bidegree $(n, n-1)$.

This yields readily the main formula for the Lefschetz number $L_{\mathrm{t}}\left(f^{\sharp}\right)$, namely

$$
L_{\mathrm{t}}\left(f^{\sharp}\right)=\sum_{p \in \operatorname{Fix}(f, \overline{\mathcal{D}})} \mu_{i}(p)-\text { p.v. } \int_{\partial \mathcal{D}} d \varphi(L) .
$$

Theorem 6.1 As defined in (4.4), the Lefschetz number of the Dolbeault complex is given by

$$
L_{\mathrm{t}}\left(f^{\sharp}\right)=\sum_{p \in \operatorname{Fix}(f, \mathcal{D})} \mu_{i}(p)+\sum_{p \in \operatorname{Fix}(f, \partial \mathcal{D})} \mu_{i}(p)+\sum_{p \in \operatorname{Fix}(f, \partial \mathcal{D})} \mu_{b}(p),
$$

where

$$
\begin{aligned}
\mu_{i}(p) & =\lim _{\varepsilon \rightarrow 0} \int_{\partial B(p, \varepsilon) \cap \mathcal{D}} \varphi(T), \\
\mu_{b}(p) & =\lim _{\varepsilon \rightarrow 0} \int_{\partial(B(p, \varepsilon) \cap \partial \mathcal{D})} \varphi(L) .
\end{aligned}
$$

Proof. By Stokes' Theorem we get

$$
\begin{aligned}
- \text { p.v. } \int_{\partial \mathcal{D}} d \varphi(L) & =-\lim _{\varepsilon \rightarrow 0} \int_{\partial \mathcal{D} \backslash O_{\varepsilon}} d \varphi(L) \\
& =\sum_{p \in \operatorname{Fix}(f, \partial \mathcal{D})} \lim _{\varepsilon \rightarrow 0} \int_{\partial(B(p, \varepsilon) \cap \partial \mathcal{D})} \varphi(L) \\
& =\sum_{p \in \operatorname{Fix}(f, \partial \mathcal{D})} \mu_{b}(p),
\end{aligned}
$$

as desired.

Theorem 6.1 gives at once the most elementary result of the Lefschetz theory. Namely, if $f$ has no fixed points then $L_{\mathrm{t}}\left(f^{\sharp}\right)=0$.

For $L_{q}$ given by (2.2) it follows from (2.1) that

$$
\begin{aligned}
\varphi(L) & =\Delta^{\sharp}(1 \times f)^{\sharp} \sum_{q=1}^{n} \frac{(n-1) !}{(2 \pi \imath)^{n}} \int_{0}^{1} \omega_{q-1}^{\prime}\left((1-\lambda) \frac{\bar{\zeta}-\bar{z}}{|\zeta-z|^{2}}+\lambda \frac{P(\zeta)}{\Phi(\zeta, z)}\right) \wedge d \zeta \\
& =\frac{(n-1) !}{(2 \pi \imath)^{n}} \int_{0}^{1} \omega^{\prime}\left((1-\lambda) \frac{\overline{\zeta-f(\zeta)}}{|\zeta-f(\zeta)|^{2}}+\lambda \frac{P(\zeta)}{\Phi(\zeta, f(\zeta))}\right) \wedge d \zeta
\end{aligned}
$$


where

$$
\left.\int_{0}^{1} F(\cdot, \lambda):=\int_{0}^{1}(d \lambda\rfloor F(\cdot, \lambda)\right) d \lambda
$$

for a differential form $F(\cdot, \lambda)$ in $\lambda$ among other variables, $d \lambda\rfloor$ being convolution by the 1 -form $d \lambda$.

\section{Evaluation of the boundary contribution}

If $p \in \partial \mathcal{D}$ is a simple fixed point of $f$ then the local index $\mu_{b}(p)$ can be explicitly calculated by a delicate but very elementary analysis. In what follows we always assume that $n>1$.

Lemma 7.1 Assume $f$ is a holomorphic map of $\mathcal{D}$ which extends to a $C^{\infty}$ map of $\overline{\mathcal{D}}$, and $p$ is a simple fixed point of $f$ on $\partial \mathcal{D}$. Then

$$
\mu_{b}(p)= \begin{cases}+\frac{1}{2} \frac{1}{\operatorname{det}_{\mathbb{C}}\left(1-f^{\prime}(p)\right)} & \text { if } p \text { is attracting } \\ -\frac{1}{2} \frac{1}{\operatorname{det}_{\mathbb{C}}\left(1-f^{\prime}(p)\right)} & \text { if } p \text { is repulsing. }\end{cases}
$$

Proof. In a small neighbourhood $U$ of $p \in \partial \mathcal{D}$ we change the coordinates by $w=\zeta-f(\zeta)$. Without loss of generality we can assume that the hypersurface $\partial \mathcal{D} \cap U$ transforms to

$$
(1-f)_{*}(\partial \mathcal{D} \cap U)=\{w: \varrho(w)<0\},
$$

where $\varrho(w)=\left|w^{\prime}\right|^{2}+\left(\Re w_{n}\right)^{2}-\Im w_{n}$ and $w^{\prime}=\left(w_{1}, \ldots, w_{n-1}\right)$, see Fig. 1 .

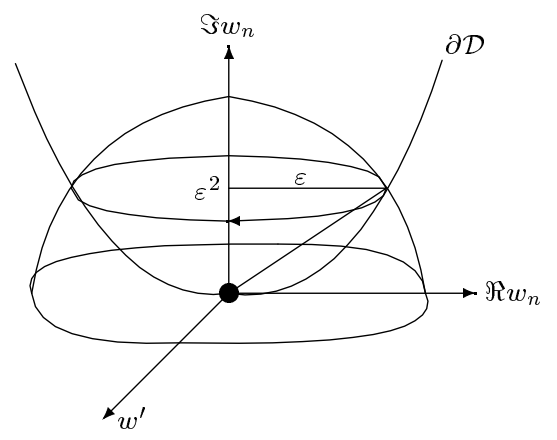

Fig. 1: Evaluation of local indices

The hypersphere $\partial B(0, \varepsilon)$ meets $(1-f)_{*}(\partial \mathcal{D} \cap U)$ in an $(2 n-2)$-dimensional sphere of radius

$$
\delta=\sqrt{-\frac{1}{2}+\sqrt{\frac{1}{4}+\varepsilon^{2}}}
$$


with centre at $\left(w^{\prime}, \Re w_{n}\right)=0$ lying in the hyperplane $\Im w_{n}=\delta^{2}$. To simplify calculation, we replace $\varepsilon$ by $\sqrt{\varepsilon^{2}+\varepsilon^{4}}$, thus reducing the intersection to the $(2 n-2)$-dimensional sphere of radius $\varepsilon$ lying in the hyperplane $\Im w_{n}=\varepsilon^{2}$. We write

$$
\mathcal{C}(\varepsilon):=\left\{w:\left|w^{\prime}\right|^{2}+\left(\Re w_{n}\right)^{2}=\varepsilon^{2}, \Im w_{n}=\varepsilon^{2}\right\}
$$

for this cycle and give it the orientation induced by the $(2 n-1)$-dimensional ball.

By definition,

$$
\mu_{b}(p)=\lim _{\varepsilon \rightarrow 0} \int_{\partial(B(p, \varepsilon) \cap \partial \mathcal{D}) \times[0,1]} \frac{(n-1) !}{(2 \pi \imath)^{n}} \omega^{\prime}(\eta(\zeta, \lambda)) \wedge d \zeta
$$

where

$$
\eta(\zeta, \lambda)=(1-\lambda) \frac{\overline{\zeta-f(\zeta)}}{|\zeta-f(\zeta)|^{2}}+\lambda \frac{P(\zeta)}{\Phi(\zeta, f(\zeta))} .
$$

After the change of variables $w=\zeta-f(\zeta)$ we get

$$
\mu_{b}(p)=\lim _{\varepsilon \rightarrow 0} \int_{\left((1-f)_{*} \partial(B(p, \varepsilon) \cap \partial \mathcal{D})\right) \times[0,1]} \frac{(n-1) !}{(2 \pi \imath)^{n}} \omega^{\prime}(\eta(\zeta(w), \lambda)) \wedge \frac{d w}{\operatorname{det}_{\mathbb{C}}\left(1-f^{\prime}(\zeta(w))\right)}
$$

with

$$
\begin{aligned}
\eta(\zeta(w), \lambda) & =(1-\lambda) \frac{\bar{w}}{|w|^{2}}+\lambda \frac{P(\zeta(w))}{\langle P(\zeta(w)), w\rangle} \\
P(\zeta(w)) & =\left(\bar{w}_{1}, \ldots, \bar{w}_{n-1}, \Re w_{n}-\frac{1}{2 \imath}\right) .
\end{aligned}
$$

Since

$$
\langle P(\zeta(w)), w\rangle=\left|w^{\prime}\right|^{2}+\left(x_{n}-\frac{1}{2 \imath}\right) w_{n}
$$

with $x_{n}=\Re w_{n}$, the components of the vector-valued function $\eta(\zeta(w), \lambda)$ are

$$
\begin{aligned}
\eta_{j} & =(1-\lambda) \frac{\bar{w}_{j}}{|w|^{2}}+\lambda \frac{2 \bar{w}_{j}}{2\left|w^{\prime}\right|^{2}+2 x_{n} w_{n}+\imath w_{n}}, \quad j=1, \ldots, n-1, \\
\eta_{n} & =(1-\lambda) \frac{\bar{w}_{n}}{|w|^{2}}+\lambda \frac{2 x_{n}+\imath}{2\left|w^{\prime}\right|^{2}+2 x_{n} w_{n}+\imath w_{n}} .
\end{aligned}
$$

For small $\varepsilon>0$ the cycle $(1-f)_{*} \partial(B(p, \varepsilon) \cap \partial \mathcal{D})$ on $(1-f)_{*}(\partial \mathcal{D} \cap U)$ is homologous to the cycle $+\mathcal{C}(\varepsilon)$, if $p$ is an attracting fixed point of $f$, and to the cycle $-\mathcal{C}(\varepsilon)$, if $p$ is a repulsing fixed point. To prove this we note that the holomorphic map $1-f$ keeps invariant the orientation of $\mathcal{D}$. Hence it keeps invariant the orientation of the tangent hyperplane at $p$ if $p$ is attracting, and changes it to the opposite one if $p$ is repulsing. By evaluating the integral over 
the surface $(1-f)_{*} \partial(B(p, \varepsilon) \cap \partial \mathcal{D})$ we may therefore replace this cycle for sufficiently small $\varepsilon>0$ by $( \pm 1) \mathcal{C}(\varepsilon)$, depending on whether $p$ is attracting or repulsing.

On every surface $\mathcal{C}(\varepsilon)$ the denominators of $\eta_{j}$ can be easily estimated, namely

$$
\begin{aligned}
2\left|w^{\prime}\right|^{2}+2 x_{n} w_{n}+\imath w_{n} & =2\left|w^{\prime}\right|^{2}+2 x_{n}^{2}+2 \imath x_{n} \Im w_{n}+\imath w_{n} \\
& =\imath x_{n}\left(1+2 \varepsilon^{2}\right)+\varepsilon^{2} \\
& \neq 0
\end{aligned}
$$

for all $\varepsilon>0$. Moreover, we have $|w|^{2}=\varepsilon^{2}+\varepsilon^{4}$.

We next transform the differential form $\omega^{\prime}(\eta(\zeta(w), \lambda)) \wedge d w$ on the surface $\mathcal{C}(\varepsilon)$. We have

$$
\begin{aligned}
\omega^{\prime}(\eta) \wedge d w & =\sum_{j=1}^{n-1}(-1)^{j-1}\left((1-\lambda) \frac{\bar{w}_{j}}{\varepsilon^{2}+\varepsilon^{4}}+\lambda \frac{2 \bar{w}_{j}}{\imath x_{n}\left(1+2 \varepsilon^{2}\right)+\varepsilon^{2}}\right) d \eta[j] \wedge d w \\
& +(-1)^{n-1}\left((1-\lambda) \frac{x_{n}-\imath \varepsilon^{2}}{\varepsilon^{2}+\varepsilon^{4}}+\lambda \frac{2 x_{n}+\imath}{\imath x_{n}\left(1+2 \varepsilon^{2}\right)+\varepsilon^{2}}\right) d \eta[n] \wedge d w .
\end{aligned}
$$

Since

$$
d w=d w^{\prime} \wedge d x_{n}
$$

holds on $\mathcal{C}$, it follows

$$
\begin{aligned}
d \eta[j] \wedge d w & =\left(\frac{1-\lambda}{\varepsilon^{2}+\varepsilon^{4}}+\frac{2 \lambda}{\imath x_{n}\left(1+2 \varepsilon^{2}\right)+\varepsilon^{2}}\right)^{n-2} d \bar{w}[j, n] \\
& \wedge\left(-\frac{x_{n}-\imath \varepsilon^{2}}{\varepsilon^{2}+\varepsilon^{4}}+\frac{2 x_{n}+\imath}{\imath x_{n}\left(1+2 \varepsilon^{2}\right)+\varepsilon^{2}}\right) d \lambda \wedge d w
\end{aligned}
$$

for $1 \leq j \leq n-1$, and

$d \eta[n] \wedge d w$

$=\left(\left(\frac{1-\lambda}{\varepsilon^{2}+\varepsilon^{4}}+\frac{2 \lambda}{\imath x_{n}\left(1+2 \varepsilon^{2}\right)+\varepsilon^{2}}\right) d \bar{w}_{1}+\bar{w}_{1}\left(\frac{-1}{\varepsilon^{2}+\varepsilon^{4}}+\frac{2}{\imath x_{n}\left(1+2 \varepsilon^{2}\right)+\varepsilon^{2}}\right) d \lambda\right)$

$\wedge \ldots$

$\wedge\left(\left(\frac{1-\lambda}{\varepsilon^{2}+\varepsilon^{4}}+\frac{2 \lambda}{\imath x_{n}\left(1+2 \varepsilon^{2}\right)+\varepsilon^{2}}\right) d \bar{w}_{n-1}+\bar{w}_{n-1}\left(\frac{-1}{\varepsilon^{2}+\varepsilon^{4}}+\frac{2}{\imath x_{n}\left(1+2 \varepsilon^{2}\right)+\varepsilon^{2}}\right) d \lambda\right)$

$\wedge d w$

whence

$$
\begin{aligned}
d \eta[n] & \wedge d w \\
& =\left(-\frac{1}{\varepsilon^{2}+\varepsilon^{4}}+\frac{2}{\imath x_{n}\left(1+2 \varepsilon^{2}\right)+\varepsilon^{2}}\right)\left(\frac{1-\lambda}{\varepsilon^{2}+\varepsilon^{4}}+\frac{2 \lambda}{\imath x_{n}\left(1+2 \varepsilon^{2}\right)+\varepsilon^{2}}\right)^{n-2} d \lambda \\
& \wedge \sum_{j=1}^{n-1}(-1)^{j-1} \bar{w}_{j} d \bar{w}[j, n] \wedge d w
\end{aligned}
$$


up to a summand which does not contain $d \lambda$.

Summarising we arrive at the equality

$$
\begin{aligned}
& \omega^{\prime}(\eta) \wedge d w \\
& =\left(\frac{1-\lambda}{\varepsilon^{2}+\varepsilon^{4}}+\frac{2 \lambda}{\imath x_{n}\left(1+2 \varepsilon^{2}\right)+\varepsilon^{2}}\right)^{n-1} d \lambda\left(-\frac{x_{n}-\imath \varepsilon^{2}}{\varepsilon^{2}+\varepsilon^{4}}+\frac{2 x_{n}+\imath}{\imath x_{n}\left(1+2 \varepsilon^{2}\right)+\varepsilon^{2}}\right) \\
& \wedge \sum_{j=1}^{n-1}(-1)^{n+j-1} \bar{w}_{j} d \bar{w}[j, n] \wedge d w \\
& +\left(\frac{1-\lambda}{\varepsilon^{2}+\varepsilon^{4}}+\frac{2 \lambda}{\imath x_{n}\left(1+2 \varepsilon^{2}\right)+\varepsilon^{2}}\right)^{n-2}\left((1-\lambda) \frac{x_{n}-\imath \varepsilon^{2}}{\varepsilon^{2}+\varepsilon^{4}}+\lambda \frac{2 x_{n}+\imath}{\imath x_{n}\left(1+2 \varepsilon^{2}\right)+\varepsilon^{2}}\right) d \lambda \\
& \wedge\left(-\frac{1}{\varepsilon^{2}+\varepsilon^{4}}+\frac{2}{\imath x_{n}\left(1+2 \varepsilon^{2}\right)+\varepsilon^{2}}\right) \sum_{j=1}^{n-1}(-1)^{n+j} \bar{w}_{j} d \bar{w}[j, n] \wedge d w
\end{aligned}
$$

which finally gives

$$
\begin{aligned}
\omega^{\prime}(\eta) \wedge d w & =\left(\frac{1-\lambda}{\varepsilon^{2}+\varepsilon^{4}}+\frac{2 \lambda}{\imath x_{n}\left(1+2 \varepsilon^{2}\right)+\varepsilon^{2}}\right)^{n-2} d \lambda \\
& \wedge \frac{\imath\left(1+2 \varepsilon^{2}\right)}{\varepsilon^{2}+\varepsilon^{4}} \frac{(-1)^{n}}{\imath x_{n}\left(1+2 \varepsilon^{2}\right)+\varepsilon^{2}} \sum_{j=1}^{n-1}(-1)^{j-1} \bar{w}_{j} d \bar{w}[j, n] \wedge d w .
\end{aligned}
$$

Using the formula

$$
\int_{0}^{1}(1-\lambda)^{k} \lambda^{n-2-k} d \lambda=\frac{k !(n-2-k) !}{(n-1) !}
$$

and the binomial formula we get

$$
\begin{aligned}
\int_{0}^{1} \omega^{\prime}(\eta) \wedge d w & =\left(\sum_{k=1}^{n-1}\left(\frac{1}{\varepsilon^{2}+\varepsilon^{4}}\right)^{k}\left(\frac{2}{\imath x_{n}\left(1+2 \varepsilon^{2}\right)+\varepsilon^{2}}\right)^{n-k}\right) \\
& \times \frac{(-1)^{n} \imath\left(1+2 \varepsilon^{2}\right)}{2(n-1)} \sum_{j=1}^{n-1}(-1)^{j-1} \bar{w}_{j} d \bar{w}[j, n] \wedge d w .
\end{aligned}
$$

Denote this $(2 n-2)$-form by $F\left(w^{\prime}, x_{n}, \varepsilon^{2}\right)$. Then

$$
\mu_{b}(p)=( \pm 1) \lim _{\varepsilon \rightarrow 0} \int_{\mathcal{C}(\varepsilon)} \frac{1}{\operatorname{det}_{\mathbb{C}}\left(1-f^{\prime}(\zeta(w))\right)} \frac{(n-1) !}{(2 \pi \imath)^{n}} F\left(w^{\prime}, x_{n}, \varepsilon^{2}\right)
$$

We pass to integration over the unit sphere $\mathbb{S}^{2 n-2}$ in the hyperplane of variables $\left(w^{\prime}, x_{n}\right)$ by replacing

$$
\begin{aligned}
w^{\prime} & \mapsto \varepsilon w^{\prime}, \\
x_{n} & \mapsto \varepsilon x_{n}
\end{aligned}
$$


for $\varepsilon>0$ small enough. Since

$$
\begin{aligned}
F\left(\varepsilon w^{\prime}, \varepsilon x_{n}, \varepsilon^{2}\right) & =\left(\sum_{k=1}^{n-1} \varepsilon^{n-1-k}\left(\frac{1}{1+\varepsilon^{2}}\right)^{k}\left(\frac{2}{\imath x_{n}\left(1+2 \varepsilon^{2}\right)+\varepsilon}\right)^{n-k}\right) \\
& \times \frac{(-1)^{n} \imath\left(1+2 \varepsilon^{2}\right)}{2(n-1)} \sum_{j=1}^{n-1}(-1)^{j-1} \bar{w}_{j} d \bar{w}[j, n] \wedge d w
\end{aligned}
$$

the most delicate summand to handle corresponds to $k=n-1$. For this summand it follows

$$
\begin{aligned}
& \lim _{\varepsilon \rightarrow 0} \int_{\mathcal{C}(1)}\left(\frac{1}{1+\varepsilon^{2}}\right)^{n-1} \frac{\imath\left(1+2 \varepsilon^{2}\right)}{\imath x_{n}\left(1+2 \varepsilon^{2}\right)+\varepsilon} \frac{(-1)^{n}}{n-1} \sum_{j=1}^{n-1}(-1)^{j-1} \bar{w}_{j} d \bar{w}[j, n] \wedge d w \\
= & \frac{1}{n-1} \lim _{\varepsilon \rightarrow 0} \int_{-1}^{1} \frac{\imath}{\imath x_{n}\left(1+2 \varepsilon^{2}\right)+\varepsilon} d x_{n} \int_{\left|w^{\prime}\right|=\sqrt{1-x_{n}^{2}}} \sum_{j=1}^{n-1}(-1)^{j-1} \bar{w}_{j} d \bar{w}[j, n] \wedge d w^{\prime} \\
= & \frac{\imath}{n-1} \lim _{\varepsilon \rightarrow 0} \int_{-1}^{1} \frac{\left(1-x_{n}^{2}\right)^{n-1}}{\imath x_{n}\left(1+2 \varepsilon^{2}\right)+\varepsilon} d x_{n} \int_{\left|w^{\prime}\right|=1} \sum_{j=1}^{n-1}(-1)^{j-1} \bar{w}_{j} d \bar{w}[j, n] \wedge d w^{\prime} \\
= & \frac{1}{2} \frac{(2 \pi \imath)^{n}}{(n-1) !}
\end{aligned}
$$

since

$$
\begin{aligned}
& \lim _{\varepsilon \rightarrow 0} \int_{-1}^{1} \frac{\left(1-x_{n}^{2}\right)^{n-1}}{\imath x_{n}\left(1+2 \varepsilon^{2}\right)+\varepsilon} d x_{n} \\
& \quad=\lim _{\varepsilon \rightarrow 0}\left(\int_{-1}^{1} \frac{\left(1-x_{n}^{2}\right)^{n-1}-1}{2 x_{n}\left(1+2 \varepsilon^{2}\right)+\varepsilon} d x_{n}+\int_{-1}^{1} \frac{1}{\imath x_{n}\left(1+2 \varepsilon^{2}\right)+\varepsilon} d x_{n}\right) \\
& =\lim _{\varepsilon \rightarrow 0}\left(\int_{-1}^{1} \frac{\left(1-x_{n}^{2}\right)^{n-1}-1}{\imath x_{n}} d x_{n}+\int_{-1}^{1} \frac{-\imath x_{n}\left(1+2 \varepsilon^{2}\right)+\varepsilon}{x_{n}^{2}\left(1+2 \varepsilon^{2}\right)^{2}+\varepsilon^{2}} d x_{n}\right) \\
& =\lim _{\varepsilon \rightarrow 0} 2 \varepsilon \int_{0}^{1} \frac{1}{x_{n}^{2}\left(1+2 \varepsilon^{2}\right)^{2}+\varepsilon^{2}} d x_{n} \\
& =\left.\lim _{\varepsilon \rightarrow 0} \frac{2}{1+2 \varepsilon^{2}}\left(\arctan \frac{1+2 \varepsilon^{2}}{\varepsilon} x_{n}\right)\right|_{0} ^{1} \\
& =\pi,
\end{aligned}
$$

the third equality being a consequence of the integrand oddness, and

$$
\int_{\left|w^{\prime}\right|=1} \sum_{j=1}^{n-1}(-1)^{j-1} \bar{w}_{j} d \bar{w}[j, n] \wedge d w^{\prime}=\int_{\left|w^{\prime}\right| \leq 1}(n-1) d \bar{w}^{\prime} \wedge d w^{\prime}
$$




$$
\begin{aligned}
& =(n-1)(2 \imath)^{n-1} \int_{\left|w^{\prime}\right| \leq 1} d v \\
& =\frac{(2 \pi \imath)^{n-1}}{(n-2) !}
\end{aligned}
$$

We next compute the limits of summands in $F\left(\varepsilon w^{\prime}, \varepsilon x_{n}, \varepsilon^{2}\right)$ corresponding to $k=1, \ldots, n-2$. To this end we proceed in much the same way as above, thus obtaining

$$
\begin{aligned}
& \lim _{\varepsilon \rightarrow 0} \int_{\mathcal{C}(1)} \varepsilon^{n-1-k}\left(\frac{1}{1+\varepsilon^{2}}\right)^{k}\left(\frac{2}{\imath x_{n}\left(1+2 \varepsilon^{2}\right)+\varepsilon}\right)^{n-k} \frac{(-1)^{n} \imath\left(1+2 \varepsilon^{2}\right)}{2(n-1)}(\ldots) \\
& =\frac{2^{n-k-1} \imath}{n-1} \frac{(2 \pi \imath)^{n-1}}{(n-2) !} \lim _{\varepsilon \rightarrow 0} \varepsilon^{n-1-k} \int_{-1}^{1} \frac{\left(1-x_{n}^{2}\right)^{n-1}}{\left(\imath x_{n}\left(1+2 \varepsilon^{2}\right)+\varepsilon\right)^{n-k}} d x_{n} \\
& =\frac{2^{n-k-1} \imath}{n-1} \frac{(2 \pi \imath)^{n-1}}{(n-2) !} \lim _{\varepsilon \rightarrow 0} \varepsilon^{n-1-k} \int_{-1}^{1} \frac{\left(1-x_{n}^{2}\right)^{n-1}}{\left(\imath x_{n}+\varepsilon\right)^{n-k}} d x_{n},
\end{aligned}
$$

(...) meaning the differential form

$$
\sum_{j=1}^{n-1}(-1)^{j-1} \bar{w}_{j} d \bar{w}[j, n] \wedge d w
$$

If $j=1, \ldots, n-1$, then

$$
\begin{aligned}
\left|\int_{-1}^{1} \varepsilon^{n-1-k} \frac{x_{n}^{2 j}}{\left(\imath x_{n}+\varepsilon\right)^{n-k}} d x_{n}\right| & \leq \int_{-1}^{1} \varepsilon^{n-1-k} \frac{\left|x_{n}\right|^{2 j}}{\left|\imath x_{n}+\varepsilon\right|^{n-k}} d x_{n} \\
& \leq \int_{-1}^{1} \varepsilon^{n-1-k} \frac{\left|x_{n}\right|^{2 j-2}}{\left|\imath x_{n}+\varepsilon\right|^{n-k-2}} d x_{n} \\
& \leq \varepsilon \int_{-1}^{1}\left|x_{n}\right|^{2 j-2} d x_{n} \\
& \rightarrow 0
\end{aligned}
$$

when $\varepsilon \rightarrow 0$. Moreover, the limit

$$
\begin{aligned}
\lim _{\varepsilon \rightarrow 0} \int_{-1}^{1} \varepsilon^{n-1-k} \frac{1}{\left(\imath x_{n}+\varepsilon\right)^{n-k}} d x_{n} & =\lim _{\varepsilon \rightarrow 0} \int_{-1 / \varepsilon}^{1 / \varepsilon} \frac{d v}{(\imath v+1)^{n-k}} \\
& =\int_{-\infty}^{\infty} \frac{d v}{(\imath v+1)^{n-k}} \\
& =0
\end{aligned}
$$


vanishes by the residue theorem.

Summarising we conclude that

$$
\begin{aligned}
\mu_{b}(p) & =( \pm 1) \lim _{\varepsilon \rightarrow 0} \int_{\mathbb{S}^{2 n-2}} \frac{1}{\operatorname{det}_{\mathbb{C}}\left(1-f^{\prime}\left(\zeta\left(\varepsilon w^{\prime}, \varepsilon x_{n}, \varepsilon^{2}\right)\right)\right)} \frac{(n-1) !}{(2 \pi \imath)^{n}} F\left(\varepsilon w^{\prime}, \varepsilon x_{n}, \varepsilon^{2}\right) \\
& =( \pm 1) \frac{1}{\operatorname{det}_{\mathbb{C}}\left(1-f^{\prime}(p)\right)} \lim _{\varepsilon \rightarrow 0} \int_{\mathbb{S}^{2 n-2}} \frac{(n-1) !}{(2 \pi \imath)^{n}} F\left(\varepsilon w^{\prime}, \varepsilon x_{n}, \varepsilon^{2}\right) \\
& =( \pm 1) \frac{1}{2} \frac{1}{\operatorname{det}_{\mathbb{C}}\left(1-f^{\prime}(p)\right)},
\end{aligned}
$$

which is due to (7.3).

It is amusing that the contributions $\mu_{i}(p)$ and $\mu_{b}(p)$ of repulsing fixed points on the boundary cancel in a wonderful way while those of attracting fixed points $p \in \partial \mathcal{D}$ are doubled. This leads to an elegant Lefschetz fixed point formula for the Dolbeault complex in the case where all fixed points of $f$ on the boundary are simple.

Corollary 7.2 Let $f$ be a holomorphic map of $\mathcal{D}$ which extends to a $C^{\infty}$ map of $\overline{\mathcal{D}}$. If all fixed points of $f$ in $\overline{\mathcal{D}}$ are simple then

$$
L_{\mathrm{t}}\left(f^{\sharp}\right)=\sum_{p \in \operatorname{Fix}(f, \mathcal{D}) \cup \mathrm{Fix}^{(a)}(f, \partial \mathcal{D})} \frac{1}{\operatorname{det}_{\mathbb{C}}(1-\partial f(p))} .
$$

Proof. Combining Theorem 6.1 and Lemmas 5.1, 5.2 and 7.1 establishes the formula.

\section{An example}

Consider a constant map $f: \overline{\mathcal{D}} \rightarrow \overline{\mathcal{D}}$, i.e., $f(z) \equiv a$ for some $a \in \overline{\mathcal{D}}$. This map has a unique fixed point $a$ in $\overline{\mathcal{D}}$, and $\operatorname{det}_{\mathbb{C}}(1-\partial f(a))=1$. In particular, $a$ is simple. If $a \in \partial \mathcal{D}$ then this fixed point is attracting, as is easy to check. The operator $f_{0}^{\sharp}$ on $\mathcal{E}(\overline{\mathcal{D}}) \cap \mathcal{O}(\mathcal{D})$ reduces to $u \mapsto u \circ f=u(a)$, i.e., to the Dirac functional supported at $a$. This operator is not of trace class, however, it acts along the constant basis function and has therefore a natural candidate for the trace, namely, $\operatorname{tr}\left(H f^{\sharp}\right)_{0}=1$. This trace can be evaluated by any representation of the Dirac functionals on the space of holomorphic functions. One can make use e.g. of the Bergman or Szegö kernels as well as of the 
Cauchy-Fantappiè integral. This agrees with our definition of the regularised trace, for

$$
\begin{aligned}
\tilde{\operatorname{tr}}\left(H f^{\sharp}\right)_{0} & =\text { p.v. } \int_{\partial \mathcal{D}} \frac{(n-1) !}{(2 \pi \imath)^{n}} \omega^{\prime}\left(\frac{P(\zeta)}{\Phi(\zeta, a)}\right) \wedge d \zeta-\int_{\mathcal{D}} \Delta^{\sharp}(1 \times f)^{\sharp} K_{S_{0}} \\
& =1 .
\end{aligned}
$$

It is immediate that $f_{q}^{\sharp}=0$ for all $q>0$. Hence it follows that $L_{\mathrm{p}}\left(f^{\sharp}\right)=0$. We thus get

$$
L_{\mathrm{t}}\left(f^{\sharp}\right)=\sum_{p \in \operatorname{Fix}(f, \mathcal{D}) \cup \operatorname{Fix}^{(a)}(f, \partial \mathcal{D})} \frac{1}{\operatorname{det}_{\mathbb{C}}(1-\partial f(p))},
$$

for both sides amount to 1 .

For a ball in $\mathbb{C}^{n}$ the operator $F_{0}$ is the Cauchy-Szegö integral. It gives an orthogonal projection onto the space of holomorphic functions in $\mathcal{D}$. Hence the regularisation

$$
\text { p.v. } \int_{\partial \mathcal{D}} K_{F_{0}}(\zeta, f(\zeta))
$$

is closely related to the Szegö projection in a ball. 


\section{References}

[AD83] L. A. Aizenberg and Sh. A. Dautov, Differential Forms Orthogonal to Holomorphic Functions or Forms, and Their Properties, AMS, Providence, R.I., 1983.

[AG83] L. Alvarez-Gaumé, Sypersymmetry and Atiyah-Singer index theorem, Commun. Math. Phys. 90 (1983), 161-173.

[Arn79] V. I. ARNOLD, Indices of singular points of 1-forms, swinging of invariants of groups generated by mappings, and singular projections of smooth surfaces, Uspekhi Mat. Nauk 34 (1979), no. 2, 3-38.

[AB67] M. F. АтіYAн and R. Bотт, A Lefschetz fixed point formula for elliptic complexes. 1, Ann. Math. 86 (1967), no. 2, 374-407.

[AS68] M. F. Atiyah and G. B. Segal, The index of elliptic operators. II, Ann. Math. 87 (1968), 531-545.

[Bis84] M. Bismut, The Atiyah-Singer theorems: a probabilistic approach. Part 2: The Lefschetz fixed point formulas, J. Funct. Anal. 57 (1984), no. 3, 329-348.

[Bis85] M. Bismut, The infinitesimal Lefschetz formulas: A heat equation proof, J. Funct. Anal. 62 (1985), 437-457.

[BdM71] L. Boutet DE Monvel, Boundary problems for pseudo-differential operators, Acta Math. 126 (1971), no. 1-2, 11-51.

[Bre88] A. V. Brenner, A fixed point theorem for the $\partial_{b}$-complex on a strictly pseudoconvex manifold, Uspekhi Mat. Nauk 43 (1988), no. 4, p. 165.

[BS81] A. V. Brenner and M. A. Shubin, The Atiyah-Bott-Lefschetz theorem for manifolds with boundary, Funkts. Analiz 15 (1981), no. 4, $67-68$.

[BS91] A. V. Brenner and M. A. Shubin, The Atiyah-Bott-Lefschetz formula for elliptic complexes on manifolds with boundary, Current Problems of Mathematics. Fundamental Directions. Vol. 38, VINITI, Moscow, 1991, pp. 119-183.

[Dol72] A. Dold, Lectures on Algebraic Topology, Springer-Verlag, Berlin et al., 1972.

[DF86] H. Donnelly and C. FefFerman, Fixed point formula for the Bergman kernel, Amer. J. Math. 108 (1986), no. 5, 1241-1258. 
[Efr88] A. V. Efremov, The Atiyah-Bott-Lefschetz formula for Hilbert bundles, Vestnik Mosk. Univ., Mat., Mekh. (1988), no. 4, 89-92.

[Eic57] M. EICHLER, Eine Verallgemeinerung der Abelschen Integrale, Math. Z. 67 (1957), 267-298.

[Fed93] B. V. Fedosov, Trace formula for Schrödinger operator, Zh. Mat. Phisiki 1 (1993), no. 4, 447-463.

[Fed00] B. Fedosov, On G-trace and G-index in deformation quantization, Lett. Math. Phys. 52 (2000), 29-49.

[Gil79] P. B. GiLKEY, Lefschetz fixed point formulas and the heat equation, Comm. Pure Appl. Math. 48 (1979), 91-147.

[Hop29] H. Hopf, Über die algebraische Anzahl von Fixpunkten, Math. Z. 29 (1929), 493-524.

[Hör85] L. Hörmander, The Analysis of Linear Partial Differential Operators. Vol. 3: Pseudo-differential operators, Springer-Verlag, Berlin et al., 1985.

[Ino82] H. InOuE, A proof of the holomorphic Lefschetz formula for higher dimensional fixed point sets by the heat equation method, J. Fac. Sci. Univ. Tokyo 29 (1982), no. 2, 267-285.

[Khe85] G. M. Khenkin, The method of integral representation in complex analysis, Current Problems of Mathematics. Fundamental Directions. Vol. 7, VINITI, Moscow, 1985, pp. 23-124.

[KN65] J. J. Kohn and L. NiRenBerG, An algebra of pseudo-differential operators, Comm. Pure Appl. Math 18 (1965), no. 1-2, 269-305.

[Kot69] T. Kotake, The fixed point theorem of Atiyah-Bott via parabolic operators, Comm. Pure Appl. Math. 22 (1969), no. 6, 789-806.

[Kyt95] A. M. Kytmanov, The Bochner-Martinelli Integral, and Its Applications, Birkhäuser Verlag, Basel et al., 1995.

[Lef26] S. Lefschetz, Intersections and transformations of complexes and manifolds, Trans. Amer. Math. Soc. 28 (1926), 1-49.

[Nes81] A. NeStKe, Cohomology of elliptic complexes and the fixed point formula of Atiyah and Bott, Math. Nachr. 94 (1981), 289-313.

[Pat73] V. K. Patodi, Holomorphic Lefschetz fixed point formula, Bull. Amer. Math. Soc. 79 (1973), 825-828. 
[Pin86] S. I. Pinchuk, Holomorphic mappings in $\mathbb{C}^{n}$ and the problem of holomorphic equivalence, Current Problems of Mathematics. Fundamental Directions. Vol. 9, VINITI, Moscow, 1985.

[dR55] G. DE Rham, Variétés différentiables. Formes, courants, formes harmoniques, Actualites Sci. Indust., Vol. 1222, Hermann, Paris, 1955 .

[See67] R. Seeley, Complex powers of an elliptic operator, Proc. Symp. Pure Math. 10 (1967), 288-307.

[Shu92] M. A. Shubin, A Lefschetz fixed point formula in reduced $L^{2}$ cohomologies on manifolds with cylinders, Symposium "Analysis on Manifolds with Singularities," Breitenbrunn, 1990. Teubner-Texte zur Mathematik 131, Teubner-Verlag, Leipzig, 1992, pp. 290-297.

[SS90] M. A. Shubin and S. Seifarth, A Lefschetz fixed point formula on manifolds with cylinder ends, C. R. Acad. Sci. Paris, Sér. 1, Math. 310 (1990), no. 12, 849-853.

[Tar95] N. N. TARkhanov, Complexes of Differential Operators, Kluwer Academic Publishers, Dordrecht, NL, 1995.

[Tol73] D. Toledo, On the Atiyah-Bott formula for isolated fixed points, J. Differential Geom. 8 (1973), no. 3, 401-436.

[TT75] D. TOledo and Y. L. TONG, Duality and intersection theory in complex manifolds. II: The holomorphic Lefschetz formula, Ann. of Math. 108 (1975), 519-538.

[Tsi92] A. K. Tsikn, Multidimensional Residues and Their Applications, AMS, Providence, RI, 1992.

[Wit82] F. Witten, Supersymmetry and Morse theory, J. Differential Geom. 17 (1982), 661-692.

(A. Kytmanov) Krasnoyarsk State University, Pr. Svobodnyi 79, 660041 Krasnoyarsk, Russia E-mail address: kytmanov@math.kgu.krasnoyarsk.su

(S. Myslivets) Krasnoyarsk State University, Pr. Svobodnyi 79, 660041 Krasnoyarsk, Russia

E-mail address: simona@math.kgu.krasnoyarsk.su

(N. Tarkhanov) Universität Potsdam, Institut für Mathematik, Postfach 6015 53, 14415 PotsDAM, GERMANY

E-mail address: tarkhanov@math.uni-potsdam.de 